\title{
NON-TRIVIAL RETARDATION EFFECTS IN DISPERSION FORCES: FROM ANOMALOUS DISTANCE DEPENDENCE TO NOVEL TRAPS
}

\author{
Johannes Fiedler* \\ Physikalisches Institut \\ Albert-Ludwigs-Universität Freiburg \\ Hermann-Herder-Str. 3 \\ 79104 Freiburg, Germany. \\ johannes.fiedler@physik.uni-freiburg.de
}

Fabian Spallek

Physikalisches Institut

Albert-Ludwigs-Universität Freiburg

Hermann-Herder-Str. 3

79104 Freiburg, Germany.

Clas Persson

Centre for Materials Science and Nanotechnology

Department of Physics

University of Oslo

P. O. Box 1048 Blindern

NO-0316 Oslo, Norway.

\author{
Kristian Berland \\ Faculty of Science and Technology \\ Norwegian University of Life Sciences, Campus Ås \\ Universitetstunet 3, NO-1430 Ås, Norway.
}

\author{
Mathias Boström* \\ Department of Energy and Process Engineering \\ Norwegian University of Science and Technology \\ NO-7491 Trondheim, Norway. \\ mathias.a.bostrom@ntnu.no
}

Iver Brevik

Department of Energy and Process Engineering Norwegian University of Science and Technology

NO-7491 Trondheim, Norway.

September 24, 2020

\begin{abstract}
In the study of dispersion forces, nonretarded, retarded and thermal asymptotes with their distinct scaling laws are regarded as cornerstone results governing interactions at different separations. Here, we show that when particles interact in a medium, the influence of retardation is qualitatively different, making it necessary to consider the non-monotonous potential in full. We discuss different regimes for several cases and find an anomalous behaviour of the retarded asymptote. It can change sign, and lead to a trapping potential.
\end{abstract}

\section{Introduction}

Dispersion forces, which include the Casimir [1] and Lifshitz [2] force between two dielectric bodies, the CasimirPolder [3] force between a dielectric body and a polarisable particle, and the London-van der Waals force [4, 5] between two polarisable particles, all arise from ground-state fluctuations of the electromagnetic fields. These forces

${ }^{*}$ Centre for Materials Science and Nanotechnology, Department of Physics, University of Oslo, P. O. Box 1048 Blindern, NO0316 Oslo, Norway 
which typically lead to an attractive interaction between the constituents, have been much studied both experimentally [6-9] and and theoretically in great detail [10-12]. Systems in which dispersion forces act across a region of vacuum have received most attention and a number of asymptotic results have been established for different geometries [13]. In most cases a simple $r^{-n}$ distance dependence for the non-retarded and thermal limits (with integer $n$ ) and an $r^{-n-1}$ distance law connecting both asymptotes in the retarded regime is found. Related to this result is the fact that the proportionality constant of the non-retarded regime is larger than that from the thermal regime. When immersed in a medium [14, 15] the interaction between particles is screened. For these cases, the retarded asymptote is often not a useful approximation for the full theory, at least not so until it merges with the thermal asymptote. To add to the complex picture we show here that the full interaction curves for London-van der Waals, Casimir-Polder and Casimir-Lifshitz in a medium are not always monotonically decaying, and they potentially have more than one extreme point. They can under certain conditions become repulsive [16,17]. Such repulsive forces can be balanced with other forces, such as buoyancy [18, 19], to stabilise a particle's position. Recently, a remarkable Lifshitz force induced trapping was experimentally observed exploiting a layered medium where short range repulsion was caused by a thin film coating, while larger distances were dominated by the underlying bulk material leading to attraction [20]. This effect was earlier theoretically predicted, e.g., by Dou et al. [21]. Here, we demonstrate that a dispersion potential alone, even in the absence of a layered surface, has the ability to trap particles without the presence of any balancing forces. In contrast to previous works, where for instance retardation effects of the Casimir force have been predicted [22] or measured [23], we demonstrate that the thermal limit is more important for medium-assisted dispersion forces. Furthermore, we demonstrate, the crossings of dielectric function also may yield a breakdown of the retarded asymptote, leading to unexpected potential behaviours.

\section{Medium-assisted Dispersion forces}

In the following, we analyse the different dispersion interactions in the presence of an environmental medium with respect to their asymptotic behaviours.

\subsection{Medium-assisted London-van-der-Waals interactions}

The van der Waals interaction is the interaction between two neutral particles, $A$ and $B$. By applying perturbation theory to the atom-field Hamiltonian [24], the energy change of the single systems can be obtained [10] (and references within)

$$
\begin{aligned}
& U_{\mathrm{vdW}}\left(\mathbf{r}_{A}, \mathbf{r}_{B}\right)=-\frac{\hbar \mu_{0}^{2}}{2 \pi} \int_{0}^{\infty} \mathrm{d} \xi \xi^{4} \\
& \quad \times \operatorname{Tr}\left[\boldsymbol{\alpha}_{A}(\mathrm{i} \xi) \cdot \mathbf{G}\left(\mathbf{r}_{A}, \mathbf{r}_{B}, \mathrm{i} \xi\right) \cdot \boldsymbol{\alpha}_{B}(\mathrm{i} \xi) \cdot \mathbf{G}\left(\mathbf{r}_{B}, \mathbf{r}_{A}, \mathrm{i} \xi\right)\right],
\end{aligned}
$$

which can be interpreted (reading from right to left) as the propagation of a virtual photon, which is created at particle $A$, of frequency i $\xi$, that propagates to particle $B\left[\mathbf{G}\left(\mathbf{r}_{B}, \mathbf{r}_{A}\right)\right]$, where it interacts with particle $B$ ( $\boldsymbol{\alpha}_{B}$ and is send back to particle $A$ followed by the interaction with it. The sum (integral) over all these exchange processes results in the van der Waals potential.

By inserting the bulk Green's function [25]

$$
\begin{aligned}
& \mathbf{G}\left(\mathbf{r}, \mathbf{r}^{\prime}, \omega\right)=-\frac{1}{3 k^{2}} \boldsymbol{\delta}(\varrho)-\frac{\mathrm{e}^{\mathrm{i} k \varrho}}{4 \pi k^{2} \varrho^{3}} \\
& \quad \times\left\{\left[1-\mathrm{i} k \varrho-(k \varrho)^{2}\right] \mathbb{I}-\left[3-3 \mathrm{i} k \varrho-(k \varrho)^{2}\right] \mathbf{e}_{\varrho} \mathbf{e}_{\varrho}\right\},
\end{aligned}
$$

with the relative coordinate $\varrho=\varrho \mathbf{e}_{\varrho}$, into Eq. (11) and applying the local-field corrections [26-28], the van der Waals potential between two particles separated by a distance $\varrho$ embedded in a medium with permittivity $\varepsilon$ reads

$$
\begin{aligned}
U_{\mathrm{vdW}}(\varrho) & =-\frac{\hbar}{16 \varepsilon_{0}^{2} \pi^{3} \varrho^{6}} \\
& \times \int_{0}^{\infty} \mathrm{d} \xi \frac{\alpha_{\mathrm{A}}^{\star}(\mathrm{i} \xi) \alpha_{\mathrm{B}}^{\star}(\mathrm{i} \xi)}{\varepsilon^{2}(\mathrm{i} \xi)} g(\xi \varrho \sqrt{\varepsilon(\mathrm{i} \xi)} / c),
\end{aligned}
$$

with

$$
g(x)=\left(3+6 x+5 x^{2}+2 x^{3}+x^{4}\right) \mathrm{e}^{-2 x} .
$$




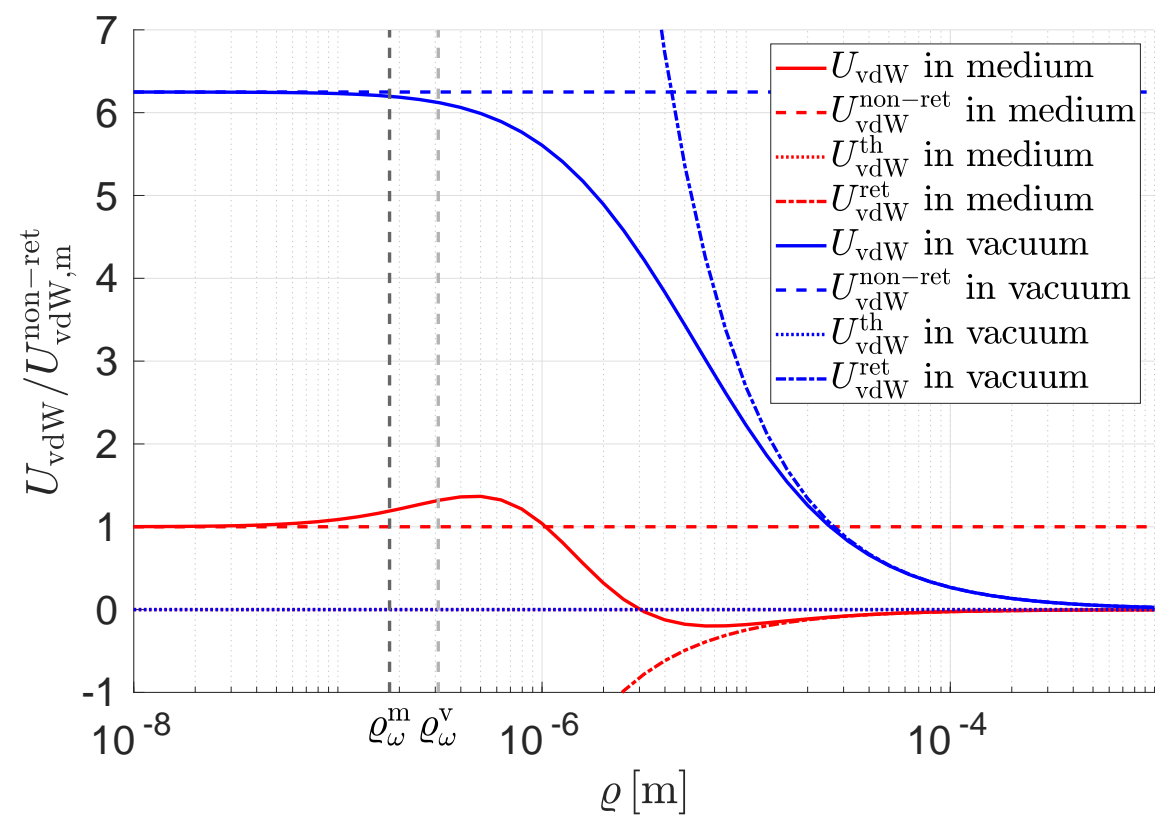

Figure 1: Van der Waals potential normalised to the medium-assisted nonretarded limit and the corresponding asymptotes (dashed lines the non-retarded limit, dashed-dotted the retarded limit and dotted lines lines the thermal limit) for a vacuum scenario (blue lines) and the corresponding case embedded in a medium (red lines).

In Eq. (3), $\alpha_{\mathrm{A}, \mathrm{B}}^{\star}$ are the environmentally screened polarisabilites. The three standard asymptotic results for the van der Waals potential [13] between two particles are as follows:

i) The non-retarded regime, in which $\varrho n(0)$ (the optical path with refractive index at zero frequency) is significantly smaller than $c / \omega_{\max }$ with $\omega_{\max }$ the largest relevant transition frequency:

$$
U_{\mathrm{vdW}}^{\mathrm{non}-\mathrm{ret}}(\varrho)=-\frac{3 \hbar}{16 \varepsilon_{0}^{2} \pi^{3} \varrho^{6}} \int_{0}^{\infty} \mathrm{d} \xi \frac{\alpha_{\mathrm{A}}^{\star}(\mathrm{i} \xi) \alpha_{\mathrm{B}}^{\star}(\mathrm{i} \xi)}{\varepsilon^{2}(\mathrm{i} \xi)}
$$

ii) the retarded regime, in which $n(0) \varrho \gg c / \omega_{\max }$ :

$$
U_{\mathrm{vdW}}^{\mathrm{ret}}(\varrho)=-\frac{23 \hbar c}{64 \varepsilon_{0}^{2} \pi^{3} \varrho^{7}} \frac{\alpha_{\mathrm{A}}^{\star}(0) \alpha_{\mathrm{B}}^{\star}(0)}{\varepsilon^{5 / 2}(0)},
$$

iii) the thermal limit for separations larger than the thermal wave length $\varrho n(0) \gg \hbar c /\left(k_{\mathrm{B}} T\right)$, which leads to

$$
U_{\mathrm{vdW}}^{\mathrm{th}}(\varrho)=-\frac{3 k_{\mathrm{B}} T}{16 \varepsilon_{0}^{2} \pi^{2} \varrho^{6}} \frac{\alpha_{\mathrm{A}}^{\star}(0) \alpha_{\mathrm{B}}^{\star}(0)}{\varepsilon^{2}(0)} .
$$

To illustrate how immersion in a medium $(\mathrm{M})$ can affect these power laws, we consider the interaction between two spherical nanoparticles of radius $a_{\mathrm{A}}$ and $a_{\mathrm{B}}$ with response functions given by single-oscillator model dielectric functions $\varepsilon_{i}(\mathrm{i} \xi)=1+\chi_{i}^{(0)} /\left[1+\left(\xi / \omega_{i}\right)^{2}\right]$ with $i=\mathrm{A}, \mathrm{B}, \mathrm{M}$ with amplitudes $\chi_{\mathrm{A}}^{(0)}=1, \chi_{\mathrm{B}}^{(0)}=4, \chi_{\mathrm{M}}^{(0)}=2$ and resonance frequencies $\omega_{\mathrm{A}}=4 \mathrm{eV}, \omega_{\mathrm{B}}=(1 / 4) \mathrm{eV}$ and $\omega_{\mathrm{M}}=(1 / 2) \mathrm{eV}$. In this model, damping effects are neglected because its impact does not influence the potential qualitatively. The corresponding polarisabilities are computed via the Clausius-Mossotti relation [29] for interactions through a void

$$
\alpha_{\mathrm{vac}}(\omega)=4 \pi a^{3} \varepsilon_{0} \frac{\varepsilon(\omega)-1}{\varepsilon(\omega)+2},
$$

and the hard-sphere model for interactions through a medium [27]

$$
\alpha_{\mathrm{HS}}(\omega)=4 \pi a^{3} \varepsilon_{0} \varepsilon_{\mathrm{M}}(\omega) \frac{\varepsilon(\omega)-\varepsilon_{\mathrm{M}}(\omega)}{\varepsilon(\omega)+2 \varepsilon_{\mathrm{M}}(\omega)},
$$




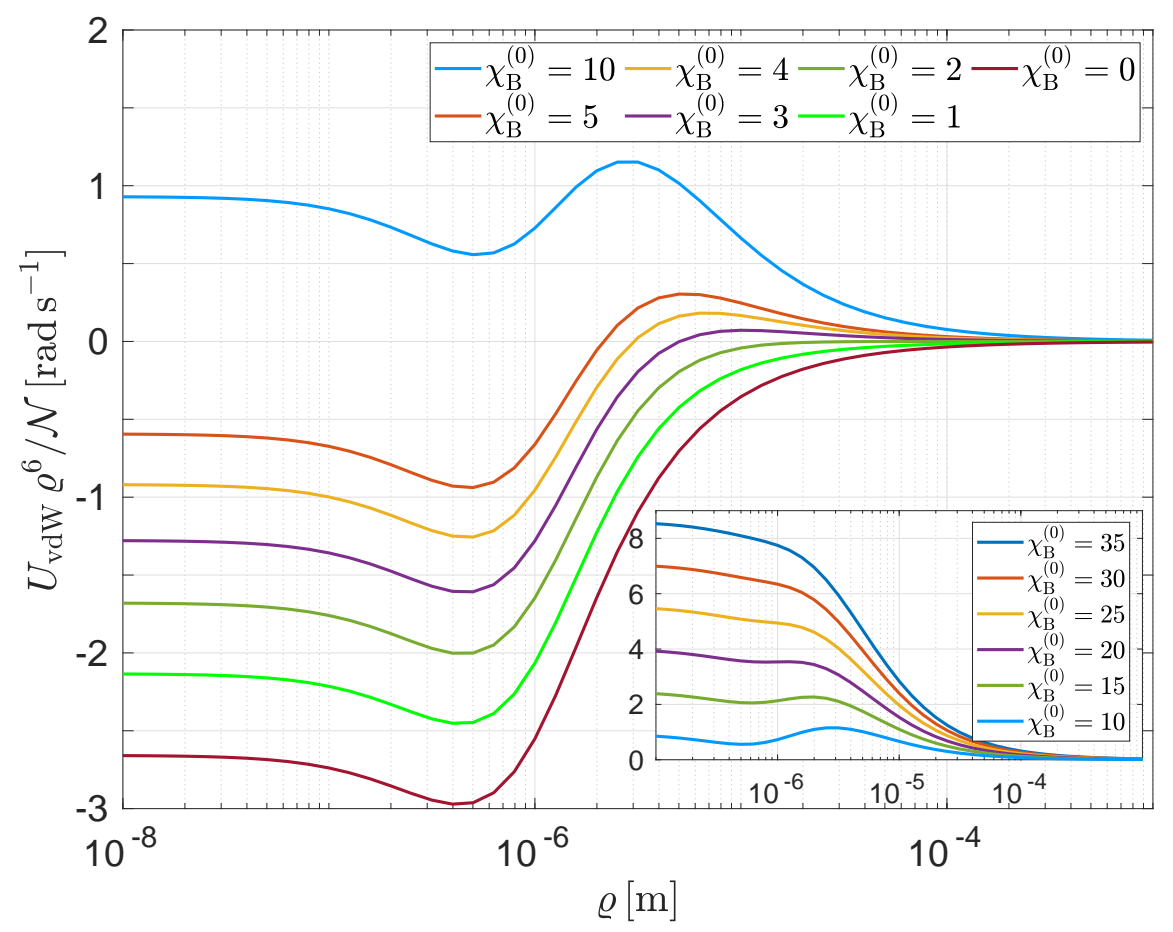

Figure 2: Dependence of the van der Waals potential by changing the dielectric's amplitude of one material with the normalisation factor $\mathcal{N}=10^{13} \hbar a_{\mathrm{A}}^{3} a_{\mathrm{B}}^{3} / \pi$. The resonances stay fixed.

with $a$ being the particle radius.

Figure 1 shows the resulting van der Waals potentials for both the vacuum and the medium-assisted cases compared with the asymptotic expressions of Eqs. (5)-(7). For clarity, the vertical axis is scaled by the sixth power of $\varrho$ ( $\left.U_{\mathrm{vdW}, \mathrm{m}}^{\text {non-ret }}=-C_{6}^{\mathrm{m}} / \varrho^{6}\right)$. In the vacuum case (blue curves), we recover the expected limits: the exact potential follows the non-retarded asymptote for small separations $\left(\varrho<\varrho_{\omega}^{\mathrm{v}}=c / \omega_{\max }\right)$; thereafter, it follows the retarded asymptotic form, until it finally matches the thermal limit whose amplitude is smaller than that of the nonretarded limit due to the steeper decrease in the retarded regime. This result is in agreement with the literature [23]. Depending on the optical response functions such, similar results can also be found in the medium-assisted case. However, if there are crossings of the dielectric functions the behaviour of the asymptotes dramatically changes. The results for the medium-assisted case (red curves) differ drastically from these vacuum findings. First of all, retardation effects kick in at significantly shorter separations, which can be attributed to the refractive index of the medium $\varrho_{\omega}^{\mathrm{m}}=c /\left[\omega_{\max } n(0)\right]$. However, it is in the intermediate regime that a truly surprising behaviour emerges. In this regime, the potential exhibits additionally distinct extreme points in the retarded regime. In fact, for this example, the retarded asymptote does not become a viable approximation for the full theory until it merges with the thermal asymptote. There is hence in effect no regime at all for which the retarded asymptote, Eq. (6) gives a good description. Finally, the prefactor of the nonretarded and thermal asymptotes differ in sign. Such potential minima have been found in earlier studies, for instance in connection with surface wetting [30]; however, here we relate this to the breakdown of the retarded regime. In Fig. 2], we explore how adjusting the amplitude of the dielectric function of particle B affects the shape of the potential with all other parameters kept fixed, as it influences the number of intersections with the dielectric functions of the medium and particles $\left(\chi_{\mathrm{B}}^{(0)}=0,1, \ldots, 10,15,20,25,30,35\right)$. It can be observed that as $\chi_{\mathrm{B}}^{(0)}$ increase, the potential changes from attraction to repulsion at short distances. For amplitudes $\chi_{\mathrm{B}}^{(0)}<2$ lower than that of the medium, only one minimum can be found according to the crossing of dielectric function of the medium and particle. For higher values a second extreme point at at larger separation occurs due to the crossing of the dielectric functions and the lower resonant frequency of particle $\left(\omega_{\mathrm{B}}\right)$ compared to that of the medium $\left(\omega_{\mathrm{M}}\right)$. For very large amplitudes $\chi_{\mathrm{B}}^{(0)}>20$, the effect of the crossings vanishes and the potential becomes smoother. 


\subsection{Medium-assisted Casimir-Polder interaction}

The anomalous asymptotic behaviour is not restricted to particle-particle interactions. For similar crossings of dielectric functions, it can also be present for a particle with polarisability $\alpha$ in front of a solid plate with permittivity $\varepsilon_{M}(i \xi)$ at temperature $T$, immersed in a liquid medium with permittivity $\varepsilon_{\mathrm{L}}(\mathrm{i} \xi)$.

The Casimir-Polder interaction, in general, describes the force between a neutral particle with polarisability $\boldsymbol{\alpha}$ in the presence of dielectric objects with permittivity $\varepsilon(\mathbf{r}, \omega)$. In analogy to the van der Waals potential, the CasimirPolder energy can be derived via the application of perturbation theory to the atom-field Hamiltonian with a single particle [31]. The result can be written as [25]

$$
U_{\mathrm{CP}}(\mathbf{r})=\frac{\hbar \mu_{0}}{2 \pi} \int_{0}^{\infty} \mathrm{d} \xi \xi^{2} \operatorname{Tr}[\boldsymbol{\alpha}(\mathrm{i} \xi) \cdot \mathbf{G}(\mathbf{r}, \mathbf{r}, \mathrm{i} \xi)] .
$$

By using the Green's function for planarly layered media [32],

$$
\begin{aligned}
\mathbf{G}\left(\mathbf{r}, \mathbf{r}^{\prime}, \omega\right)= & \frac{\mathrm{i}}{8 \pi^{2}} \int \frac{\mathrm{d}^{2} k^{\|}}{k_{1}^{\perp}} \mathrm{e}^{\mathrm{ik} \mathbf{k}^{\|} \cdot\left(\mathbf{r}-\mathbf{r}^{\prime}\right)+\mathrm{i} k_{1}^{\perp}\left(z+z^{\prime}\right)} \\
& \times \sum_{\sigma=s, p} r_{\sigma}^{12} \mathbf{e}_{\sigma+}^{1} \mathbf{e}_{\sigma-}^{1},
\end{aligned}
$$

with the reflection coefficients

$$
r_{s}^{12}=\frac{k_{1}^{\perp}-k_{2}^{\perp}}{k_{1}^{\perp}+k_{2}^{\perp}}, \quad r_{p}^{12}=\frac{\varepsilon_{2} k_{1}^{\perp}-\varepsilon_{1} k_{2}^{\perp}}{\varepsilon_{2} k_{1}^{\perp}+\varepsilon_{1} k_{2}^{\perp}},
$$

again applying the local-field corrections for the excess polarisability, and introducing the temperature dependence according to the standard substitution for the integral to the Matsubara sum

$$
\begin{aligned}
& 2 \pi \hbar \int \mathrm{d} \xi f(\xi) \mapsto k_{\mathrm{B}} T \sum_{n=0}^{\infty}{ }^{\prime} f\left(\xi_{n}\right) \\
& =k_{\mathrm{B}} T\left[\frac{1}{2} f\left(\xi_{0}\right)+\sum_{n=1}^{\infty} f\left(\xi_{n}\right)\right],
\end{aligned}
$$

with respect to the non-resonant part of the Casimir-Polder potential [33], with the primed sum denotes a sum over Matsubara frequencies $\xi_{n}=2 \pi n k_{\mathrm{B}} T / \hbar$, with the first term weighed by $1 / 2$ [10], the Casimir-Polder potential is given by [10]

$$
\begin{aligned}
& U_{\mathrm{CP}}\left(z_{\mathrm{A}}\right)=\frac{k_{\mathrm{B}} T \mu_{0}}{4 \pi} \sum_{n=0}^{\infty}{ }^{\prime} \alpha^{\star}\left(\mathrm{i} \xi_{n}\right) \xi_{n}^{2} \\
& \times \int_{0}^{\infty} \mathrm{d} k \frac{k}{\kappa_{\mathrm{L}}^{\perp}} \mathrm{e}^{-2 \kappa_{\mathrm{L}}^{\perp} z_{\mathrm{A}}}\left[\frac{\kappa_{\mathrm{L}}^{\perp}-\kappa_{\mathrm{M}}^{\perp}}{\kappa_{\mathrm{L}}^{\perp}+\kappa_{\mathrm{M}}^{\perp}}\right. \\
& \left.\quad-\left(1+2 \frac{\kappa_{\mathrm{L}}^{\perp} c^{2}}{\xi_{n}^{2} \varepsilon_{\mathrm{L}}(\mathrm{i} \xi)}\right) \frac{\varepsilon_{\mathrm{M}} \kappa_{\mathrm{L}}^{\perp}-\varepsilon_{\mathrm{L}} \kappa_{\mathrm{M}}^{\perp}}{\varepsilon_{\mathrm{M}} \kappa_{\mathrm{L}}^{\perp}+\varepsilon_{\mathrm{L}} \kappa_{\mathrm{M}}^{\perp}}\right],
\end{aligned}
$$

with the imaginary part of the perpendicular wave vectors given by $\kappa_{i}^{\perp}=\sqrt{\varepsilon_{i}(\mathrm{i} \xi) \xi^{2} / c^{2}+k^{2}}$. It takes the following forms in the non-retarded asymptote

$$
U_{\mathrm{CP}}^{\mathrm{non}-\mathrm{ret}}(z)=-\frac{C_{3}}{z^{3}}
$$

with

$$
C_{3}=\frac{k_{\mathrm{B}} T}{8 \pi \varepsilon_{0}} \sum_{n=0}^{\infty}, \frac{\alpha^{\star}\left(\mathrm{i} \xi_{n}\right)}{\varepsilon_{\mathrm{L}}\left(\mathrm{i} \xi_{n}\right)} \frac{\varepsilon_{\mathrm{M}}\left(\mathrm{i} \xi_{n}\right)-\varepsilon_{\mathrm{L}}\left(\mathrm{i} \xi_{n}\right)}{\varepsilon_{\mathrm{M}}\left(\mathrm{i} \xi_{n}\right)+\varepsilon_{\mathrm{L}}\left(\mathrm{i} \xi_{n}\right)},
$$

and the retarded asymptote

$$
U_{\mathrm{CP}}^{\mathrm{ret}}(z)=-\frac{C_{4}}{z^{4}}
$$


A PREPRINT - SEPTEMBER 24, 2020

\begin{tabular}{cccccccc}
\hline Mol. & $\mathrm{C}_{3}$ & $\mathrm{C}_{4}$ & $\mathrm{C}_{3 \mathrm{~T}}$ & $\mathrm{Z}_{\text {trap }}$ & $z_{0}$ & $z_{\omega}$ & $\omega_{\text {trap }}$ \\
\hline $\mathrm{CH}_{4}$ & -45.9 & 11638 & 31.3 & 26.0 & 59.2 & 1.0 & 6.5 \\
$\mathrm{CO}$ & 109.9 & 12755 & 34.3 & - & 0.8 & 0.2 & - \\
$\mathrm{CO}_{2}$ & 31.3 & 15464 & 41.6 & - & 2.4 & 0.3 & - \\
$\mathrm{H}_{2} \mathrm{~S}$ & -189.9 & 12958 & 34.8 & 132.1 & 142.1 & 2.2 & 0.1 \\
$\mathrm{~N}_{2}$ & 66.4 & 11181 & 30.1 & - & 1.5 & 0.3 & - \\
$\mathrm{NO}_{2}$ & -1.3 & 14466 & 38.9 & 1.0 & 3.6 & 0.3 & 3930 \\
$\mathrm{~N}_{2} \mathrm{O}$ & -66.8 & 14574 & 39.2 & 21.0 & 41.4 & 0.5 & 7.8 \\
$\mathrm{O}_{2}$ & 96.4 & 10903 & 29.3 & - & 1.1 & 0.2 & - \\
$\mathrm{O}_{3}$ & -24.9 & 14562 & 39.2 & 7.2 & 26.0 & 0.3 & 90.8 \\
\hline
\end{tabular}

Table 1: Data of $C_{3}$-coefficients $\left(\left[\mu \mathrm{eV}(\mathrm{nm})^{3}\right]\right.$, eq. $\left.(16)\right), C_{4}$-coefficients $\left(\left[\mu \mathrm{eV}(\mathrm{nm})^{4}\right]\right.$, eq. $\left.(18)\right), C_{3 \mathrm{~T}}$-coefficients $\left(\left[\mu \mathrm{eV}(\mathrm{nm})^{3}\right]\right.$, eq. $\left.(20)\right)$, trapping distances $\left(\mathrm{z}_{\text {trap }}[\mathrm{nm}]\right)$, and the corresponding trapping frequency $\left(\omega_{\text {trap }}[\mathrm{MHz}]\right)$ for different molecules dissolved in water at $T=273.16 \mathrm{~K}$ near a water-air surface. Positive $C_{3}, C_{4}$, and $C_{3 \mathrm{~T}}$ values correspond to attraction. Further, the distances for the impact of retardation $\left(z_{\omega}[\mathrm{nm}]\right)$ are given for each case. The thermal effects occur on distances larger than $z_{\mathrm{T}}=893 \mathrm{~nm}$. As an approximation of the trapping distance tabled the values for $z_{0}$ that corresponds to the roots of the polarisabilities.

with

$$
\begin{aligned}
C_{4}= & \frac{3 \hbar c \alpha^{\star}(0)}{64 \pi^{2} \varepsilon_{0} \varepsilon_{\mathrm{L}}^{3 / 2}(0)} \int_{1}^{\infty} \mathrm{d} v \\
& \times\left[\left(\frac{2}{v^{2}}-\frac{1}{v^{4}}\right) \frac{\varepsilon_{\mathrm{M}} \sqrt{\varepsilon_{\mathrm{L}}} v-\varepsilon_{\mathrm{L}} \sqrt{\varepsilon_{\mathrm{L}}\left(v^{2}-1\right)+\varepsilon_{\mathrm{M}}}}{\varepsilon_{\mathrm{M}} \sqrt{\varepsilon_{\mathrm{L}}} v+\varepsilon_{\mathrm{L}} \sqrt{\varepsilon_{\mathrm{L}}\left(v^{2}-1\right)+\varepsilon_{\mathrm{M}}}}\right. \\
& \left.-\frac{1}{v^{4}} \frac{\sqrt{\varepsilon_{\mathrm{L}}} v-\sqrt{\varepsilon_{\mathrm{L}}\left(v^{2}-1\right)+\varepsilon_{\mathrm{M}}}}{\sqrt{\varepsilon_{\mathrm{L}}} v+\sqrt{\varepsilon_{\mathrm{L}}\left(v^{2}-1\right)+\varepsilon_{\mathrm{M}}}}\right],
\end{aligned}
$$

and finally, in the thermal limit, it is given by

$$
U_{\mathrm{CP}}^{\mathrm{th}}(z)=-\frac{C_{3 \mathrm{~T}}}{z^{3}}
$$

with

$$
C_{3 \mathrm{~T}}=-\frac{k_{\mathrm{B}} T}{16 \pi \varepsilon_{0}} \frac{\alpha^{\star}(0)}{\varepsilon_{\mathrm{L}}(0)} \frac{\varepsilon_{\mathrm{M}}(0)-\varepsilon_{\mathrm{L}}(0)}{\varepsilon_{\mathrm{M}}(0)+\varepsilon_{\mathrm{L}}(0)} .
$$

The behaviour of the three asymptotes in vacuum is similar to the previously discussed van der Waals case depicted in Fig. 1 with modified power laws ( $r^{-3}$ for nonretarded, $r^{-4}$ for retarded and $r^{-3}$ for the thermal asymptote). Further details can be found in Ref. [34].

Applying this model to a specific example, we consider greenhouse gases with polarisabilities and particle and cavity radii taken from Ref. [27]. The gases immersed in water interact with the water-air interface. Further, we use the finite-size model of Refs. [26, 27] to describe the excess polarisability that arises when taking into account the finitesize effects of the particles and a vacuum layer surrounding the particle to avoid the contact between particle and solvent [35]. The calculated potentials and the corresponding asymptotes are depicted in Fig. 3. The corresponding parameters for the asympotes, traping distances and frequencies are given in Table 1 It can be observed that hydrogen sulfide $\left(z_{\text {trap }}=132 \mathrm{~nm}\right)$, methane $(26 \mathrm{~nm})$, nitrogen dioxide $(21 \mathrm{~nm})$, ozone $(7 \mathrm{~nm})$ and nitrous oxide $(1 \mathrm{~nm})$ are trapped near the surface. For these systems, a potential minimum occurs between the non-retarded and thermal limits and the retarded limit does not appear as an $r^{-4}$ power law. Some correlation between the trapping distance $z_{\text {trap }}$ and the position corresponding to the change in sign of dielectric function $z_{0}=c /\left[\omega_{0} n(0)\right]$ with $\alpha\left(\mathrm{i} \omega_{0}\right)=0$ can be found, as well as between the trapping distance and the transition distance between the retarded and non-retarded limit $z_{\omega}$. However, a simple correlation between one of them alone and the trapping distances cannot be expected, because the potential depends on all three quantities: the dielectric functions of the medium and the polarisabilities of the particles, whereas each of these parameters only depends on one of them. The gas molecule $\mathrm{H}_{2} \mathrm{~S}$ deviates from most other gas molecules in the non-retarded limit in having a very large negative $\mathrm{C}_{3}$ in Table 1 . One would have expected $\mathrm{H}_{2} \mathrm{~S}$ to be hydrophobic as was recently discussed in Ref. [36]. This result will require further investigation.

By comparing the depth of the potential minimum with the thermal energy one finds that the stability of the trap is given at temperatures far below the freezing temperature of water. This means that for these examples, the interactions 


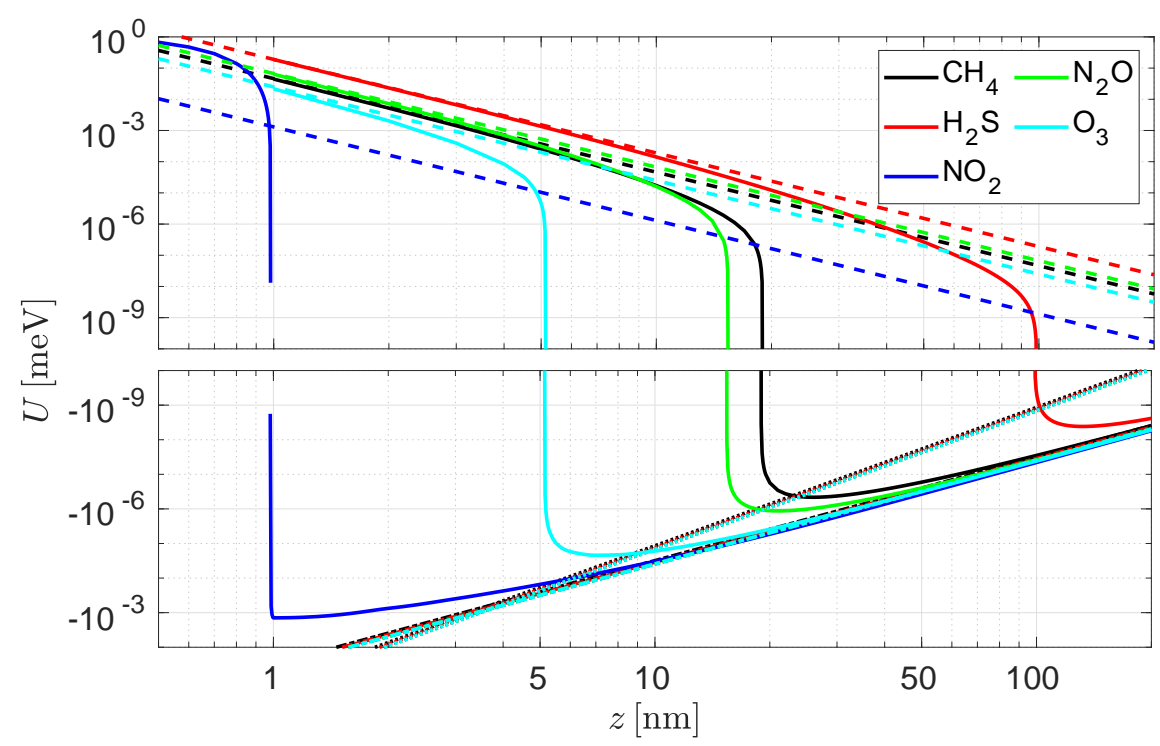

Figure 3: The Casimir-Polder potential for dissolved $\mathrm{CH}_{4}$ molecule (black), $\mathrm{H}_{2} \mathrm{~S}$ (red), $\mathrm{NO}_{2}$ (blue), $\mathrm{N}_{2} \mathrm{O}$ (green) and $\mathrm{O}_{3}$ (cyan) near water surface shown together with the two limiting asymptotes (non-retarded dashed), and retarded (dotted).

are not strong enough to trap particles. In terms of particles dissolved in a medium, one might be able to discern a slightly higher concentration of particles at these specific trapping distances. We note that the thermal stability of the trap increases with decreasing trap distance. Further, the energetic minimum of the potential becomes steeper with decreasing trap distance resulting in a narrowed trapping potential the closer it gets to the interface. For different materials these trends will be similar.

Single gas molecules reveal anomalous interactions near a water surface, but the typical energy minimum is not sufficiently deep to act as an effective trap. Inspired by the observed Casimir-Polder repulsion for air bubbles in water near solid surfaces [16, 37] we consider a system where we expect trapping could occur. Specifically, we consider an air bubble dissolved in liquid bromobenzene [17] in front of an horizontal, or vertical, amorphous silica surface. For modelling, we used parameters corresponding to amorphous silica (Volume of the $\mathrm{SiO}_{2}$ unit: $V_{\mathrm{v}}=41.14 \AA^{3}$ [38]) at room temperature $(T=298 \mathrm{~K})$. The air bubble can, due to a crossing of dielectric functions for amorphous silica and bromobenzene at a specific frequency, be trapped, via short range repulsive and long range attractive Casimir-Lifshitz forces [10]. Here, we apply a simple version of the Derjaguin approximation to estimate the force on the sphere of radius $R, f_{\text {sphere }}(x)=2 \pi R U_{\text {plane }}(x)$ with $U_{\text {plane }}(x)$ being the energy for planar system. We then integrate the force from infinity up to the specific distance $x$, to obtain the interaction free energy acting on a sphere at a distance $x$ from the planar interface. The minimum sphere radius $R_{\min }$ will be estimated from the size that provides a trapping energy larger or equal to $k_{\mathrm{B}} T$. Here, we find an estimate for a thermally stable position of approximately $7 \mathrm{~nm}$ in front of the surface for bubbles with a radius much larger than $R_{\min }=200 \mathrm{~nm}$. Hence, we are in a range for relative sizes and distances where the Derjaguin approximation is appropriate to use, but where surface roughness and various other nanoscale effects are important. The short range repulsion experienced by the typical air bubble is expected to lead to low surface friction. We note that for bubbles, below or above a surface, there will also be some influence on trapping distance from buoyancy, $b=V g\left(\rho_{\mathrm{l}}-\rho_{\mathrm{a}}\right)$, which depends on volume $(V)$, gravitational constant $g$ and the difference in mass density for bubble $\rho_{\mathrm{a}}$ and the liquid $\rho_{\mathrm{l}}$. However, in front of a vertical surface buoyancy only acts to move the air bubble upwards while the proposed mechanism keeps it moving with low friction at a fixed trapping distance from the surface.

\section{Application: tuneable trap}

Ideally, a larger trapping distance than found above would be more realistic for experimental realisation. Thus, to introduce a scalable parameter for tuning the trap, we consider in our final example a two-component fluid surrounding the particle. For the dielectric functions of mixtures between fluid $1\left(\varepsilon_{1}\right)$ in fluid $2\left(\varepsilon_{2}\right)$ we use a Lorentz-Lorenz model [19, 39, 40], where we introduce the volume fraction $p$ of fluid 1 in fluid 2 . We chose the liquids bromobenzene and methanol in front of a polystyrene surface [17] as the dielectric function of the latter lies between both fluids. An 


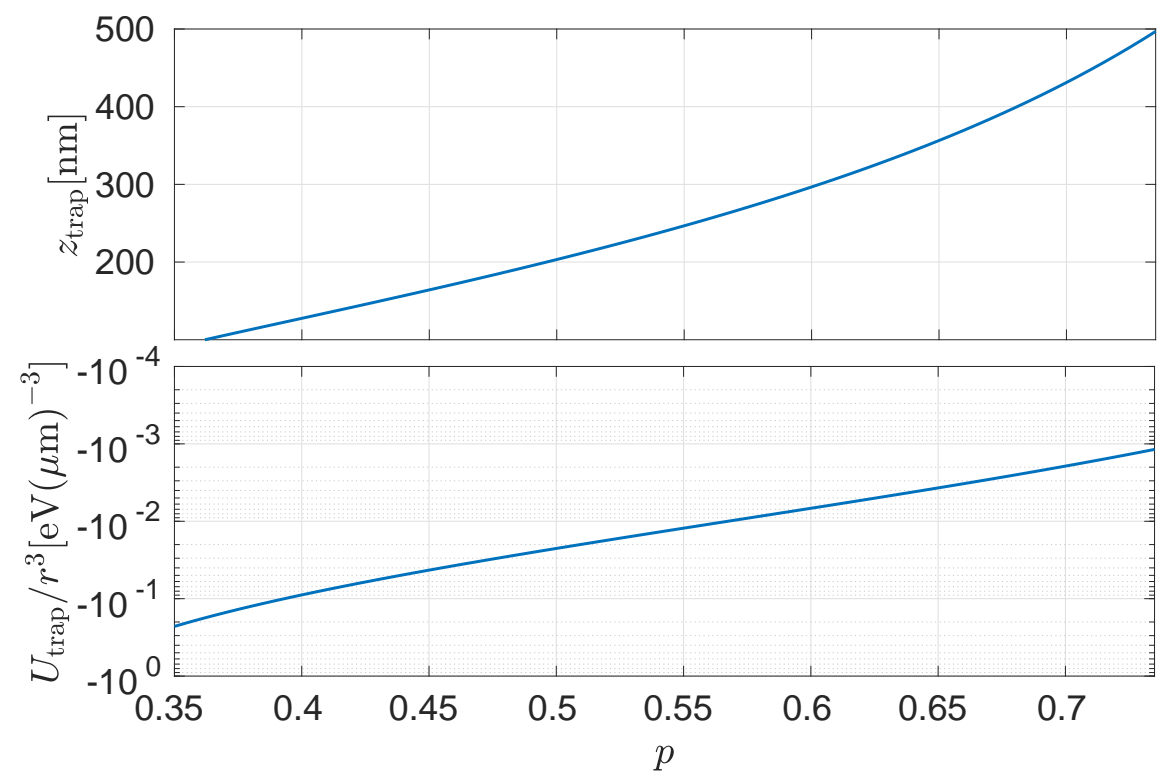

Figure 4: Trap parameters depending on the volume fraction $p$.

illustration of the resulting dielectric functions can be found in Ref. [41]. In this case, the crossings of the dielectric functions depend on the volume fraction. We used the example of an anatase- $\mathrm{TiO}_{2}$ spherical nanoparticle whose dielectric function was taken from Ref. [42]. As we consider larger particles compared to few-atomic molecules from the example above, we describe the excess polarisability via the hard-sphere model as in the introductory van der Waals example. The resulting parameters for the trap are given in Fig. [4 We predict that the trapping can be tuned over a wide range of distances $(100-500 \mathrm{~nm})$ by changing the liquid mixture.

\section{Results and Conclusion}

To conclude, we have shown that the impact of retardation dramatically changes the asymptotic behaviour of dispersion interactions in media. In contrast to the ordinary theory in vacuum, the retarded power laws are not applicable and a consideration of the full interaction potential is required instead. Further, the transition distance between nonretarded and retarded regimes strongly decreases by immersing the interacting particles in a liquid due to its refractive index. This extends previous work on Casimir-Lifshitz forces in fluids (see e.g. Refs. [2, 17, 19, 20, 23, 30, 43]) to a more general case where retarded dispersion forces can reveal a very complex behaviour in media. For this reason, considerations of retardation effects are important for medium-assisted dispersion force experiments, for example Casimir experiments [16, 23, 43]; medium-assisted optical tweezers [44]; colloidal systems [45]; and in future measurements of the Casimir torque in a medium [46,47]. Beyond these impacts, we have shown that the near-field effect of dispersion forces in colloidal system yields an inhomogeneous particle density distribution near interfaces due to trapping potentials. The introduced mechanism can for instance be used to trap nanoparticles at low temperature, specifically nanodiamonds [48], by choosing liquid nitrogen, liquid fluorine, or atomic clouds as an environmental medium. The presented theory is more general and can be applied to several systems beyond Casimir experiments, especially medium-assisted spectroscopy in nanodroplets [49-51].

\section{Acknowledgments}

We acknowledge support from the Research Council of Norway (Project 250346). We gratefully acknowledge support by the German Research Council (grant BU 1803/6-1, S.Y.B. and J.F., BU 1803/3-1, S.Y.B. and F.S.).

\section{References}

[1] H. B. G. Casimir. K. Ned. Akad. Wet., 51:793, 1948. 
[2] I.E. Dzyaloshinskii, E.M. Lifshitz, and L.P. Pitaevskii. The general theory of van der Waals forces. Advances in Physics, 10(38):165-209, 1961.

[3] H. B. G. Casimir and D. Polder. The influence of retardation on the London-van der Waals forces. Phys. Rev., 73:360-372, 1948.

[4] F. London. Zur Theorie und Systematik der Molekularkräfte. Zeitschrift für Physik, 63(3):245-279, Mar 1930.

[5] Johannes Diderik van der Waals. Over de Continuiteit van den Gas-en Vloeistoftoestand. Dissertation, University Leiden, 1873.

[6] Daniel Ebeling, Marina Šekutor, Marvin Stiefermann, Jalmar Tschakert, Jeremy E. P. Dahl, Robert M. K. Carlson, André Schirmeisen, and Peter R. Schreiner. London Dispersion Directs On-Surface Self-Assembly of [121]Tetramantane Molecules. ACS Nano, 11(9):9459-9466, 2017.

[7] W. Schöllkopf and J. P. Toennies. Nondestructive Mass selection of Small Van der Waals Clusters. Science, 266:1345-1348, 1994.

[8] R. E. Grisenti, W. Schöllkopf, J. P. Toennies, G. C. Hegerfeldt, and T. Köhler. Determination of Atom-Surface Van der Waals Potentials from Transmission-Grating Diffraction Intensities. Phys. Rev. Lett., 83:1755-1758, 1999.

[9] M. Arndt, O. Nairz, J. Vos-Andreae, C. Keller, G. van der Zouw, and A. Zeilinger. Wave-particle duality of C60 molecules. Nature, 401(6754):680-682, 1999.

[10] S. Y. Buhmann. Dispersion Forces I: Macroscopic quantum electrodynamics and ground-state Casimir, CasimirPolder and van der Waals forces. Springer Tracts in Modern Physics. Springer, Heidelberg, 2012.

[11] Diego Dalvit, Peter Milonni, David Roberts, and Felipe da Rosa. Casimir Physics. Lecture Notes in Physics. Springer, 2011.

[12] Nicolas Cherroret, Romain Guerout, Astrid Lambrecht, and Serge Reynaud. Statistical approach to CasimirPolder potentials in heterogeneous media. Phys. Rev. A, 92(4), 2015.

[13] Stefan Yoshi Buhmann, Stefan Scheel, and James Babington. Universal Scaling Laws for Dispersion Interactions. Phys. Rev. Lett., 104:070404, Feb 2010.

[14] D. S. Ether, L. B. Pires, S. Umrath, D. Martinez, Y. Ayala, B. Pontes, G. R. de S. Araújo, S. Frases, G.-L. Ingold, F. S. S. Rosa, N. B. Viana, H. M. Nussenzveig, and P. A. Maia Neto. Probing the Casimir force with optical tweezers. EPL (Europhysics Letters), 112(4):44001, nov 2015.

[15] Peter Loskill, Hendrik Hähl, Thomas Faidt, Samuel Grandthyll, Frank Müller, and Karin Jacobs. Is adhesion superficial? Silicon wafers as a model system to study van der Waals interactions. Advances in Colloid and Interface Science, 179-182:107 - 113, 2012.

[16] Rico F. Tabor, Rogerio Manica, Derek Y. C. Chan, Franz Grieser, and Raymond R. Dagastine. Repulsive van der Waals Forces in Soft Matter: Why Bubbles Do Not Stick to Walls. Phys. Rev. Lett., 106:064501, Feb 2011.

[17] P. J. van Zwol and G. Palasantzas. Repulsive Casimir forces between solid materials with high-refractive-index intervening liquids. Phys. Rev. A, 81:062502, Jun 2010.

[18] P. Thiyam, J. Fiedler, S. Y. Buhmann, C. Persson, I. Brevik, M. Boström, and D. F. Parsons. Ice Particles Sink below the Water Surface Due to a Balance of Salt, van der Waals, and Buoyancy Forces. The Journal of Physical Chemistry C, 122(27):15311-15317, 2018.

[19] Mathias Boström, Maofeng Dou, Oleksandr I. Malyi, Prachi Parashar, Drew F. Parsons, Iver Brevik, and Clas Persson. Fluid-sensitive nanoscale switching with quantum levitation controlled by $\alpha$-Sn/ $\beta$-Sn phase transition. Phys. Rev. B, 97:125421, Mar 2018.

[20] Rongkuo Zhao, Lin Li, Sui Yang, Wei Bao, Yang Xia, Paul Ashby, Yuan Wang, and Xiang Zhang. Stable Casimir equilibria and quantum trapping. Science, 364(6444):984-987, 2019.

[21] Maofeng Dou, Fei Lou, Mathias Boström, Iver Brevik, and Clas Persson. Casimir quantum levitation tuned by means of material properties and geometries. Phys. Rev. B, 89:201407, May 2014.

[22] Mathias Boström, Simen Å. Ellingsen, Iver Brevik, Drew F. Parsons, and Bo E. Sernelius. Sign of the casimirpolder interaction between atoms and oil-water interfaces: Subtle dependence on dielectric properties. Phys. Rev. A, 85:064501, Jun 2012.

[23] Michael A. Bevan and Dennis C. Prieve. Direct measurement of retarded van der waals attraction. Langmuir, 15(23):7925-7936, 1999.

[24] Hassan Safari, Stefan Yoshi Buhmann, Dirk-Gunnar Welsch, and Ho Trung Dung. Body-assisted van der waals interaction between two atoms. Phys. Rev. A, 74:042101, Oct 2006. 
[25] S. Scheel and S.Y. Buhmann. Macroscopic quantum electrodynamics - Concepts and applications. Act. Phys. Slov., 58(5):675 - 809, 2008.

[26] Agnes Sambale, Stefan Yoshi Buhmann, Dirk-Gunnar Welsch, and Marin-Slodoban Tomaš. Local-field correction to one- and two-atom van der Waals interactions. Phys. Rev. A, 75:042109, Apr 2007.

[27] Johannes Fiedler, Priyadarshini Thiyam, Anurag Kurumbail, Friedrich A. Burger, Michael Walter, Clas Persson, Iver Brevik, Drew F. Parsons, Mathias Boström, and Stefan Y. Buhmann. Effective Polarizability Models. The Journal of Physical Chemistry A, 121(51):9742-9751, 2017.

[28] Friedrich Anton Burger, Johannes Fiedler, and Stefan Yoshi Buhmann. Zero-point electromagnetic stress tensor for studying Casimir forces on colloidal particles in media. EPL, 121(2):24004, 2018.

[29] John David Jackson. Classical electrodynamics. Wiley, New York, NY, 3rd ed. edition, 1999.

[30] Michael Elbaum and M. Schick. Application of the theory of dispersion forces to the surface melting of ice. Phys. Rev. Lett., 66:1713-1716, Apr 1991.

[31] S Y Buhmann, Ho Trung Dung, and D-G Welsch. The van der waals energy of atomic systems near absorbing and dispersing bodies. Journal of Optics B: Quantum and Semiclassical Optics, 6(3):S127-S135, mar 2004.

[32] M. S. Tomaš. Green function for multilayers: Light scattering in planar cavities. Phys. Rev. A, 51:2545-2559, Mar 1995.

[33] S Y Buhmann. Dispersion Forces II: Many-Body Effects, Excited Atoms, Finite Temperature and Quantum Friction. Springer Tracts in Modern Physics. Springer, 2012.

[34] Stefan Yoshi Buhmann, Stefan Scheel, Simen Å. Ellingsen, Klaus Hornberger, and Andreas Jacob. CasimirPolder interaction of fullerene molecules with surfaces. Phys. Rev. A, 85:042513, Apr 2012.

[35] Alexander Held and Michael Walter. Simplified continuum solvent model with a smooth cavity based on volumetric data. The Journal of Chemical Physics, 141(17):174108, 2014.

[36] Johannes Fiedler, Mathias Boström, Clas Persson, Iver Brevik, Robert Corkery, Stefan Yoshi Buhmann, and Drew F. Parsons. Full-spectrum high-resolution modeling of the dielectric function of water. The Journal of Physical Chemistry B, 124(15):3103-3113, 2020.

[37] V. Esteso, S. Carretero-Palacios, P. Thiyam, H. Miguez, D. F. Parsons, I. Brevik, and M. Boström. Trapping of Gas Bubbles in Water at a Finite Distance below a Water-Solid Interface. Langmuir, 35(12):4218-4223, 2019.

[38] Oleksandr I. Malyi, Mathias Boström, Vadym V. Kulish, Priyadarshini Thiyam, Drew F. Parsons, and Clas Persson. Volume dependence of the dielectric properties of amorphous SiO2. Phys. Chem. Chem. Phys, 18(10):74837489, 2016.

[39] D. E. Aspnes. Local-field effects and effective-medium theory: A microscopic perspective. American Journal of Physics, 50(8):704-709, 1982.

[40] Mathias Boström, Robert W. Corkery, Eduardo R. A. Lima, Oleksandr I. Malyi, Stefan Y. Buhmann, Clas Persson, Iver Brevik, Drew F. Parsons, and Johannes Fiedler. Dispersion Forces Stabilize Ice Coatings at Certain Gas Hydrate Interfaces That Prevent Water Wetting. ACS Earth and Space Chemistry, 3(6):1014-1022, 2019.

[41] Johannes Fiedler, Clas Persson, and Stefan Yoshi Buhmann. Spectroscopy of Nanoparticles without Light. Phys. Rev. Applied, 13:014025, Jan 2020.

[42] Maofeng Dou and Clas Persson. Comparative study of rutile and anatase $\mathrm{SnO} 2$ and $\mathrm{TiO} 2$ : Band-edge structures, dielectric functions, and polaron effects. Journal of Applied Physics, 113(8):083703, 2013.

[43] J. N. Munday, Federico Capasso, V. Adrian Parsegian, and Sergey M. Bezrukov. Measurements of the CasimirLifshitz force in fluids: The effect of electrostatic forces and Debye screening. Phys. Rev. A, 78:032109, Sep 2008.

[44] Dongliang Gao, Weiqiang Ding, Manuel Nieto-Vesperinas, Xumin Ding, Mahdy Rahman, Tianhang Zhang, ChweeTeck Lim, and Cheng-Wei Qiu. Optical manipulation from the microscale to the nanoscale: fundamentals, advances and prospects. Light: Science \& Applications, 6(9):e17039-e17039, 2017.

[45] Georges Brügger, Luis S Froufe-Pérez, Frank Scheffold, and Juan José Sáenz. Controlling dispersion forces between small particles with artificially created random light fields. Nature Communications, 6(1):7460, 2015.

[46] David A. T. Somers and Jeremy N. Munday. Casimir-Lifshitz Torque Enhancement by Retardation and Intervening Dielectrics. Phys. Rev. Lett., 119:183001, Nov 2017.

[47] P. Thiyam, Prachi Parashar, K. V. Shajesh, O. I. Malyi, M. Boström, K. A. Milton, I. Brevik, J. Forsman, and C. Persson. Effect of excess charge carriers and fluid medium on the magnitude and sign of the Casimir-Lifshitz torque. Phys. Rev. B, 100:205403, Nov 2019. 
[48] Yannick Dumeige, Jean-François Roch, Fabien Bretenaker, Thierry Debuisschert, Victor Acosta, Christoph Becher, Georgios Chatzidrosos, Arne Wickenbrock, Lykourgos Bougas, Alexander Wilzewski, and Dmitry Budker. Infrared laser threshold magnetometry with a NV doped diamond intracavity etalon. Opt. Express, 27(2):1706-1717, Jan 2019.

[49] Matthieu Dvorak, Markus Müller, Tobias Knoblauch, Oliver Bünermann, Alexandre Rydlo, Stefan Minniberger, Wolfgang Harbich, and Frank Stienkemeier. Spectroscopy of 3, 4, 9, 10-perylenetetracarboxylic dianhydride (PTCDA) attached to rare gas samples: Clusters vs. bulk matrices. I. Absorption spectroscopy. The Journal of Chemical Physics, 137(16):164301, 2012.

[50] Michael Kelbg, Andreas Heidenreich, Lev Kazak, Michael Zabel, Bennet Krebs, Karl-Heinz Meiwes-Broer, and Josef Tiggesbäumker. Comparison of Electron and Ion Emission from xenon Cluster-Induced Ignition of Helium Nanodroplets. The Journal of Physical Chemistry A, 122(41):8107-8113, 2018.

[51] Oliver Stauffert, Sharareh Izadnia, Frank Stienkemeier, and Michael Walter. Optical signatures of pentacene in soft rare-gas environments. The Journal of Chemical Physics, 150(24):244703, 2019. 